\title{
Técnica gráfico - plástica para mejorar la creatividad en estudiantes de instituciones unidocentes
}

\author{
Jenny Aparicio Crisanto \\ martha_castillo_noriega@hotmail.com \\ https://orcid.org/0000-0002-2167-2530 \\ Universidad César Vallejo, Piura - Perú \\ César Balladares Atoche \\ cesarballadares@hotmail.com \\ https://orcid.org/0000-0001-8242-7742 \\ Universidad César Vallejo, Piura - Perú
}

\section{RESUMEN}

El propósito es demostrar que la aplicación de la técnica gráfico - plástica mejora la creatividad en estudiantes de instituciones unidocentes - nivel inicial, UGEL Paita - 2021. En relación a la metodología del trabajo investigativo se optó por el paradigma positivista, además de priorizar el enfoque cuantitativo, el tipo de investigación es aplicada, con un diseño pre experimental, esto utilizando el prestest y postest. Para el recojo de los datos se utilizó la técnica de la observación y como instrumento la lista de cotejo, además la muestra estuvo constituida por 28 estudiantes de las instituciones educativas unidocentes del nivel inicial de la UGEL Paita, es oportuno indicar que los instrumentos fueron validados por juicio de expertos y pasaron fiabilidad Alfa de Cronbach, en ambos casos con resultados positivos. Los resultados determinaron que la aplicación de la técnica gráfico - plástica mejora la creatividad, esto se manifiesta en el control y mejora de la fluidez, originalidad, flexibilidad y elaboración en lo que corresponde a la creatividad. Frente a estos resultados se aceptó la hipótesis de investigación, es decir que la aplicación de la técnica gráfico - plástica influye significativamente en la creatividad.

Palabras clave: creatividad; originalidad; fluidez; gráfico-plástico. 


\title{
Graphic-plastic technique to improve creativity in students of single-teacher institutions
}

\begin{abstract}
The purpose is to demonstrate that the application of the graphic-plastic technique improves creativity in students from single-teacher institutions - initial level, UGEL Paita - 2021. Regarding the methodology of the investigative work, the positivist paradigm was chosen, in addition to prioritizing the approach quantitative, the type of research is applied, with a pre-experimental design, this using the pretest and posttest. To collect the data, the observation technique was used and the checklist was used as an instrument; in addition, the sample was made up of 28 students from the unified educational institutions of the initial level of the UGEL Paita, it is appropriate to indicate that the instruments were validated by expert judgment and passed Cronbach's alpha reliability, in both cases with positive results. The results determined that the application of the graphic - plastic technique improves creativity, this is manifested in the control and improvement of fluidity, originality, flexibility and elaboration in what corresponds to creativity. Faced with these results, the research hypothesis was accepted, that is to say that the application of the graphic - plastic technique significantly influences creativity.
\end{abstract}

Keywords: creativity; originality; fluency; graphic-plastic.

Artículo recibido: 30 noviembre. 2021 Aceptado para publicación: 29 diciembre 2021 Correspondencia: cmarlinpano@gmail.com Conflictos de Interés: Ninguna que declarar 


\section{INTRODUCCIÓN}

La creatividad, en estos dos últimos años justamente en circunstancias de la pandemia se ha manifestado de diversas formas en los niños, en tal sentido, se ha ostentado en los hogares al generar, articular o aplicar ideas, las mismas que surgen de la espontaneidad en cada niño de las instituciones educativas y seguramente en aquellos que tampoco asisten a una institución educativa; sin embargo, lo realizan en su rutina diaria dentro de sus hogares. En tal sentido, UNICEF (2020) para el contexto de Panamá considera que se debe "incentivar a los niños a dibujar, esta estrategia mejora y desarrolla la escritura y la creatividad", en donde se destaca la autoconfianza y sobre todo permite mostrar sus sentimientos y emociones frente a la diversidad que se presenta en el espacio que lo rodea. En Perú el Ministerio de Educación del Perú desde el año 2016, ha puesto de manifiesto un conjunto de estrategias y capacitaciones a los docentes para promover la creatividad en los estudiantes, esto facilita que puedan imaginar escenarios y sobre todo poder resolver problemáticas que se presenten en su experiencia diaria, por otro lado en el documento oficial del programa curricular se precisan objetivos fundamentales para desarrollar la creatividad en la cual se establece "Crea proyectos artísticos que demuestran habilidades artísticas iniciales para comunicar ideas, sentimientos, observaciones y experiencias".

En Piura Gutiérrez (2021) considera que la creatividad en los niños es fundamental, pero que se logrará con la labor que deben asumir los padres y maestros, despertando y promoviendo de manera espontánea; además se considera que debemos vincularnos con nuestras habilidades con la finalidad de fantasear, improvisar y sobre todo crear de una manera espontánea y libre, demostrando manejo de emociones, seguridad y flexibilidad producto de la niñez. A nivel de UGEL Paita, hoy en día se trabaja poco la creatividad en las aulas del nivel inicial, las docentes se dedican en gran mayoría a entregar materiales diseñados y que el estudiante repite o traza en compañía de algún familiar, en consecuencia, se repiten la indicación del docente, en este sentido no se considera la creatividad de los niños toda vez que no permiten hacer otras actividades ajenas a la indicación planteada.

Asimismo, es importante conocer investigaciones en otros contextos que tenga relación con el presente informe, en tal sentido según la búsqueda bibliográfica tenemos antecedentes internacionales, como es el caso de Beteta (2017) en su estudio Diseño de 
un programa de técnicas gráfico plásticas para el desarrollo de la motricidad fina en los niños de la I.E. $N^{\circ}$ 088, trabajó con una población de 104 estudiantes y una muestra de 56 estudiantes, aplicando pre y post test. Se demostró que el programa de Técnicas Gráfico Plásticas beneficia positivamente e igualmente a toda la población a la que se le aplique. De acuerdo al autor de este estudio, la aplicación de las técnicas grafico plásticas, ayudan a mejorar la creatividad a quienes las reciben, demostrando de esta manera que la aplicación de éstas trajo como resultado mejorar la creatividad en los estudiantes.

De la misma manera, Parra (2016) realizó una investigación sobre la creatividad en la educación de infantes, aplicado en servicios educativos públicos y privados de la ciudad de Bucaramanga-Universidad de Granada. Este estudio se desarrolló con muestreo aleatorio por conglomerados. El tipo de investigación es descriptiva con una metodología descriptiva comparada donde se realizará un Estudio analógico. Los instrumentos que emplearon fue el test CREA. Las conclusiones fueron: La creatividad en infantes de 7 a 10 años de edad, quienes cursan asignaturas en instituciones públicas o privadas del municipio de Bucaramanga, no se encuentran determinadas por la edad, es decir la correlación obtenida entre la variable edad de los infantes de los colegios de los cuatro núcleos educativos del municipio de Bucaramanga y el resultado logrado en el test CREA, es significativo, relevante, siendo una correlación menos significativa infiriéndose que la variable edad no es causal en el nivel de creatividad, en los estudiantes.

En lo que se refiere al trabajo investigativo, sobre la variable creatividad se sustenta en la teoría de las inteligencias múltiples, también con un corte cognitivo (Gardner, 1998) el autor plantea, que es múltiple la creatividad al igual que la inteligencia, y argumenta los test, pruebas de creatividad fracasan porque suponen que desempeñarse en forma óptima al utilizar el pensamiento divergente, garantizan el pronóstico de comportamiento creativo futuro en cualquier otro contexto. Asimismo, según el autor, el pacto faustiano apoya el nivel de desarrollo de la creatividad, es decir las personas renunciamos a todo lo cómodo, atractivo, para lograr un objetivo preciso, determinado, concluyendo, que la creatividad transmite, otorga el aspecto de motivación para lograr metas concretas.

Además de lo descrito en el acápite anterior, las teorías sobre la variable creatividad tenemos teoría de la creatividad de Guilford en palabras de Sternberg, Lubart. (1997) argumenta que la creatividad es un proceso en el cual es parte del pensar divergente. Debemos considerar que el pensar creativamente se sostiene por los mismos procesos 
mentales que el pensamiento cognitivo, los procedimientos son: codificar, comparar, procesamiento de analizar y sintetizar, etc. Observándose que todas las personas tienen ambas formas de pensar, en general no todos utilizan las capacidades creativas e invertir el dominio de un pensamiento sobre otro. Para Sawyer (2016) considera que desarrollar las capacidades creativas implican proporcionar, acceder, motivar, permitir la accesibilidad de ambas formas de pensar, logrando desarrollar la capacidad de acudir a ambos, haciéndolo funcional el procedimiento de crear. Generalmente, se tiende a relacionar la capacidad creativa con el pensar de forma innovadora, apartándose de un estilo de pensamiento habitual, rutinario. Actualmente, las investigaciones, han demostrado que la capacidad creativa emerge de una interrelación de ambos estilos de pensamiento.

Asimismo, tenemos el aporte de Piaget (1960) el cual considera que los infantes son creativos innatos. El juego es la estrategia que permite el desarrollo de pensar abstractamente. Los niños al terminar el periodo de juego simbólico empiezan a desarrollar el pensamiento creativo (Swift, 2015). Al principio los niños empiezan a reproducir contextos cotidianos y los repiten, este proceso ayuda a transitar del pensamiento concreto al pensamiento abstracto. Por lo antes mencionado, los docentes deben propiciar espacios con condiciones que promuevan lograr desarrollar el pensamiento creativo desde edades tempranas. Es evidente, que todas las personas nacemos con potencial para desarrollar la creatividad, pues nadie con ella, esto se logra relacionando las capacidades de crear, expresar, comunicar. La familia es el primer contexto donde se desarrolla el niño, en segundo lugar, tenemos el contexto de las instituciones educativas, ambos contextos deben orientar, guiar, promover y desarrollar la creatividad (Sternberg, Lubart, 1997). El segundo contexto debe mantener una comunicación permanente con la familia para acompañar de forma óptima el desarrollo de la creatividad en los niños.

Diversos autores conceptualizan que la creatividad es algo traspasa el método científico riguroso hacia contextos menos rígidos, que permiten crear, innovar ideas. Olivares (2014) estableciendo que la capacidad creativa es un procedimiento que presenta una dificultad al cerebro con claridad, para luego "innovar, crear, producir ideas, conceptos, nociones, o esquemas en contextos nuevos o no convencional, es decir, que se manifiesta 
la capacidad de imaginar, inventar, resolución de problemas en los aspectos probados" (p. 9).

De acuerdo a lo escrito anteriormente se destaca la importancia de la creatividad, en este aspecto es vital el conocimiento de las dimensiones que se abordan para poder evaluar el nivel del mismo en los estudiantes, es por ello que se revisó científicamente el aporte de Beaudot (2016), el cual considera las siguientes dimensiones de la creatividad: a. Fluidez: es la dimensión que estudia la habilidad organizar elevados números de conceptos respecto a un contenido delimitado. La forma de potenciar la capacidad creativa en el contexto del colegio, por ejemplo: pedir al niño la relación entre acontecimientos, palabras, eventos además en palabras de Perdomo (2017) sostiene que la fluidez es la cantidad de opiniones y procedimientos que tiene el niño para solucionar un problema en un determinado tiempo., es por ello que concluye que mientras más ideas lleguen a su cabeza, más fluidez tiene. b. Flexibilidad: es un criterio de la capacidad creativa: transformar el procedimiento para lograr la resolución del problema. Se origina en la habilidad de afrontar problemas desde diferentes contextos; asimismo consideramos el aporte de Flores (2017) quienes la conceptualizan como "la destreza para concebir diversos géneros los cuales son susceptibles de cambio o variación de acuerdo al contexto incluso se considera como habilidad para acomodarse, transformar conductas y asumir nuevos puntos de vista” (p, 9);

Además, también se considera la originalidad como dimensión, que implica proposiciones, procedimientos, productos de forma única o diversa. Se producen respuestas innovadoras o poco frecuentes. Dentro de la escuela se promueve al estimular nuevas teorías en los estudiantes propone; asimismo consideramos el aporte de Flores (2017) en el cual considera que la originalidad es la producción de ideas inusuales estadísticamente infrecuentes, es decir una aportación brillante de carácter único la cual sorprende y contiene valores nuevos. Es la conceptualización que fundamenta lo innovador con pericia para observar el contexto desde una representación novedosa convirtiéndola en insuperables y por ende novedosa; d. Elaboración: es el criterio que permite observar el nivel de complemento, perfeccionamiento o complejo de la idea creativa. Para fomentar en la clase, se le presenta al estudiante una ilustración de historia con dibujos en los cuales se percatan detalles (Beaudot, 2016). 
En consecuencia, de acuerdo a lo anterior, se considera la habilidad de generar representaciones, las cuales se realizan a través del proceso de planeamiento, desarrollo y ejecución de proyectos. El criterio de elaboración es la forma, condición que transforma la fórmula en solución y acción decisiva, así como la exigencia del impulso creativo a su realización. (Larraz, 2021)

Por otro lado, en lo que concierne a la técnica grafica gráfico - plástica, tenemos que Bermúdez y Perreros (2011) observan a las técnicas gráfico plásticas como estrategias que fortalecen el desarrollo y la actividad comunicativa de las personas en general, manifestándose de diversas formas; pero todas, por medio de recursos reutilizables, plástico, arcilla, madera, papel, cartón, etc. Estas tienen una característica fundamental el que promueven el aspecto creativo innato del niño, la que lo impulsa a encajar con el contexto del otro.

Asimismo para Díaz (2006), las técnicas gráfico plásticas desarrollan en el estudiante la capacidad de comunicar lo que siente y lo que piensa; ya que estas técnicas desembocan en actividades donde los niños no solamente observan, sin embargo, también, piensan, sienten y actúan. Asimismo, según García (2012), citado por Mestanza (2016), las técnicas grafo plásticas permiten la libre expresión del espíritu humano, favoreciendo el instinto creador del niño, al proponer variadas y ricas actividades en las que el estudiante hará uso de su imaginación y libre albedrío para superar el reto que le proponga su docente.

Por lo descrito se puede inferir que el desarrollar las capacidades creativas implican proporcionar, acceder, motivar, permitir la accesibilidad de ambas formas de pensar, logrando desarrollar la capacidad de acudir a ambos, haciéndolo funcional el procedimiento de crear. Generalmente, se tiende a relacionar la capacidad creativa con el pensar de forma innovadora, apartándose de un estilo de pensamiento habitual, rutinario, asimismo actualmente, las investigaciones, han demostrado que la capacidad creativa emerge de una interrelación de ambos estilos de pensamiento.

Por lo antes expuesto se consideró la siguiente interrogante de investigación: ¿En qué medida la técnica gráfico - plástica mejora la creatividad en estudiantes de instituciones unidocentes - nivel inicial, UGEL Paita - 2021? Además, el presente estudio de investigación tuvo como objetivo general: Demostrar que la aplicación de la técnica gráfico - plástica mejora la creatividad en estudiantes de instituciones unidocentes - nivel 
inicial, UGEL Paita - 2021. Como objetivos específicos tenemos: Determinar que la aplicación de la técnica gráfico - plástica mejora la fluidez en estudiantes; Demostrar que la aplicación de la técnica gráfico - plástica mejora la originalidad en estudiantes; Determinar que la aplicación de la técnica gráfico - plástica mejora la flexibilidad en estudiantes; y Demostrar que la aplicación de la técnica gráfico - plástica mejora la elaboración en estudiantes; de instituciones unidocentes - nivel inicial, UGEL Paita 2021.

\section{ESTRATEGIAS METODOLÓGICAS O MATERIALES Y MÉTODOS}

De acuerdo con las características de la investigación, el presente informe es aplicado, también llamada constructiva o utilitaria. En tal sentido Sánchez y Reyes (1998, p.13-14) sostienen que se "caracteriza por su interés en la aplicación de los conocimientos teóricos a determinada situación concreta y las consecuencias prácticas que de ella se deriven". Asimismo, el diseño es pre- experimental, administrándole pre test a un solo grupo, para luego administrar el tratamiento o estímulo, que consistió en quince sesiones de aprendizaje, después de ello, aplicar el post test o medición posterior.

Para el recojo de los datos se utilizó como técnica la observación, dada la edad cronológica de los niños; la misma que consistió en registrar en forma sistemática y confiable el comportamiento y conducta manifestada por los sujetos. De la misma manera se utilizó la guía de observación, que según Carrasco (2009), “nos permite recoger información para analizar y determinar indicadores que estrictamente dependen del problema y objetivo de la investigación", la misma que fue construida para medir el nivel de creatividad con sus dimensiones: fluidez, originalidad, flexibilidad, elaboración. Estos instrumentos fueron validados por juicios de expertos, los mismos que son profesionales probos en la materia de investigación, concluyendo validez de contenido aceptable; además se utilizó el coeficiente Alfa de Cronbach, para determinar la fiabilidad del instrumento el mismo que fue de 0,839 .

Es oportuno mencionar que la muestra estuvo constituida por 28 niños que pertenecen a las instituciones unidocentes de la jurisdicción de la Ugel Paita; esta muestra de estudio fue elegido de manera no probabilística a criterio del investigador. (Ary et al., 1989). Por otro lado, en relación al procesamiento de los datos se utilizó la estadística descriptiva e inferencial, la primera para promediar los valores de la variable y las dimensiones; 
además la segunda se utilizó para validar o rechazar la hipótesis, en este sentido se desarrolló la prueba paramétricas T-Student para muestras relacionadas.

\section{RESULTADOS Y DISCUSIÓN}

Los resultados fueron obtenidos con la aplicación de la lista de cotejo de forma presencial a pesar de las circunstancias por la pandemia, lógicamente las preguntas están orientadas a la consecución del objetivo general, que de acuerdo al recojo de los datos se obtuvo los siguientes resultados:

\section{Tabla 1}

Niveles de la variable creatividad

\begin{tabular}{ccccc}
\hline & \multicolumn{2}{c}{ Pre Test } & \multicolumn{2}{c}{ Post Test } \\
\hline ALTO & fi & $\%$ & fi & $\%$ \\
MEDIO & 0 & 0 & 28 & 100 \\
BAJO & 28 & 100 & 0 & 0 \\
TOTAL & 0 & 0 & 0 & 0 \\
\hline
\end{tabular}

Nota: Niveles de creatividad del pretest y postest mediante la lista de cotejo.

Tal como se manifiesta en la Tabla 1, se aprecia que el 100\% se encuentran en nivel medio en el pre test y luego se aprecia que el 100\% en ubica en nivel alto en el post test. Entonces se determina que la estrategia gráfico - plástica mejora la creatividad, esto se manifiesta debido a que los estudiantes generan ideas, son innovadores, crean formas, inclusive tienen capacidad de adaptación, modifican ideas y las desarrollan.

\section{Tabla 2}

Niveles de la dimensión fluidez.

\begin{tabular}{ccccc}
\hline & \multicolumn{3}{c}{ Pre Test } & Post Test \\
\hline ALTO & fi & $\%$ & fi & $\%$ \\
MEDIO & 2 & 7.14 & 28 & 100 \\
BAJO & 7 & 67,86 & 0 & 0 \\
TOTAL & 28 & 25 & 0 & 0 \\
\end{tabular}

Nota: Niveles de la dimensión fluidez del pretest y postest mediante la lista de cotejo.

En la tabla 2 se aprecia que el 67,86\% se ubica en nivel medio, el 25\% en el nivel bajo, en el pre test; y luego se aprecia que el $100 \%$ en ubica en nivel alto en el post test. 
Entonces se determina que la estrategia gráfico - plástica mejora la dimensión fluidez. En este sentido se descubre los efectos que se producen al combinar un material con otro, muestra y comenta de forma espontánea, realiza al crear proyectos utilizando diversos materiales, asimismo muestra sus creaciones y observa las creaciones de otros.

\section{Tabla 3}

Niveles de la dimensión originalidad

\begin{tabular}{ccccc}
\hline & & Pre Test & \multicolumn{2}{c}{ Post Test } \\
\hline ALTO & fi & $\%$ & fi & $\%$ \\
MEDIO & 26 & 0 & 28 & 100 \\
BAJO & 2 & 7,14 & 0 & 0 \\
TOTAL & $\mathbf{2 8}$ & $\mathbf{1 0 0}$ & 0 & 0
\end{tabular}

Nota: Niveles de la dimensión originalidad del pretest y postest mediante la lista de cotejo.

En la tabla 3 se aprecia que el $92,86 \%$ se encuentran en nivel medio en el pre test y luego se aprecia que el $100 \%$ en ubica en nivel alto en el post test. Entonces se determina que la estrategia gráfico - plástica mejora la originalidad.

\section{Tabla 4}

\section{Niveles de la dimensión flexibilidad}

\begin{tabular}{|c|c|c|c|c|}
\hline & \multicolumn{2}{|c|}{ Pre Test } & \multicolumn{2}{|c|}{ Post Test } \\
\hline & fi & $\%$ & fi & $\%$ \\
\hline ALTO & 0 & 0 & 28 & 100 \\
\hline MEDIO & 21 & 75 & 0 & 0 \\
\hline BAJO & 7 & 25 & 0 & 0 \\
\hline TOTAL & 28 & 100 & 28 & 100 \\
\hline
\end{tabular}

Nota: Niveles de la dimensión flexibilidad del pretest y postest mediante la lista de cotejo.

En la tabla 4, se aprecia que el 75\% está en nivel medio, el 25\% nivel bajo, en el pre test; y luego se aprecia que el 100\% en ubica en nivel alto en el post test. Entonces se determina que la estrategia gráfico - plástica mejora la flexibilidad. 
Tabla 5 Niveles de la dimensión elaboración

\begin{tabular}{ccccc}
\hline & \multicolumn{2}{c}{ Pre Test } & \multicolumn{2}{c}{ Post Test } \\
\hline ALTO & $\mathrm{f}$ & $\%$ & $\mathrm{fi}$ & $\%$ \\
MEDIO & 0 & 0 & 27 & 96,42 \\
BAJO & 27 & 96,42 & 1 & 3,58 \\
TOTAL & 1 & 3,58 & 0 & 0 \\
& 28 & 100 & 28 & 100 \\
\hline
\end{tabular}

Nota: Niveles de la dimensión elaboración del pretest y postest mediante la lista de cotejo.

En la tabla 5 se aprecia que el $96,42 \%$ se encuentran en nivel medio en el pre test y luego se aprecia que el 96,42\% en ubica en nivel alto en el post test. Quedando aun el 3,58\% en nivel medio: Se puede sostener que la estrategia gráfico - plástica mejora sustancialmente la elaboración.

Tabla 8 Prueba de hipótesis general

\section{Estadísticas de muestra única}

\begin{tabular}{lcccc}
\hline & N & Media & $\begin{array}{c}\text { Desviación } \\
\text { estándar }\end{array}$ & $\begin{array}{c}\text { Media de } \\
\text { error estándar }\end{array}$ \\
\hline Creatividad Pre test & 28 & 24,57 & 1,136 & 0,215 \\
Creatividad Post test & 28 & 38,75 & 1,11 & 0,21 \\
\hline
\end{tabular}

Tabla 9 Prueba de hipótesis especificas

\section{Prueba de muestras emparejadas}

\begin{tabular}{|c|c|c|c|c|c|c|c|c|c|}
\hline & \multicolumn{5}{|c|}{ Diferencias emparejadas } & \multirow[t]{3}{*}{$\mathrm{t}$} & \multirow[t]{3}{*}{ gl } & \multirow[t]{3}{*}{$\begin{array}{c}\text { Sig. } \\
\text { (bilateral) }\end{array}$} \\
\hline & & \multirow[t]{2}{*}{ Media } & \multirow[t]{2}{*}{$\begin{array}{l}\text { Desviación } \\
\text { estándar }\end{array}$} & \multirow{2}{*}{$\begin{array}{l}\text { Media } \\
\text { de error } \\
\text { estándar }\end{array}$} & \multicolumn{2}{|c|}{$\begin{array}{c}95 \% \text { de intervalo } \\
\text { de confianza de la } \\
\text { diferencia }\end{array}$} & & & \\
\hline & & & & & Inferior & Superior & & & \\
\hline Par 1 & $\begin{array}{l}\text { Fluidez_Posteest - } \\
\text { Fluidez_Pretest }\end{array}$ & 2,964 & ,793 & ,150 & 2,657 & 3,272 & 19,789 & 27 & ,000 \\
\hline Par2 & $\begin{array}{l}\text { Originaliadd_Postest - } \\
\text { Originalidad_Pretest }\end{array}$ & 4,964 & ,744 &, 141 & 4,676 & 5,253 & 35,285 & 27 & ,000 \\
\hline Par 3 & $\begin{array}{l}\text { Flexibilidad_Postest - } \\
\text { Flexibilidad_Pretest }\end{array}$ & 2,929 & ,663 & ,125 & 2,672 & 3,186 & 23,384 & 27 & ,000 \\
\hline Par 4 & $\begin{array}{l}\text { Elaboración_Postest - } \\
\text { Elaboración_Pretest }\end{array}$ & 3,321 & ,905 & ,171 & 2,971 & 3,672 & 19,423 & 27 & ,000 \\
\hline
\end{tabular}

Nota: De acuerdo a los resultados se puede evidenciar que $\mathrm{p}<0.05$ en tal sentido se rechazan las $\mathrm{H}_{0}$; es decir existe diferencia estadísticamente significativa entre el puntaje promedio obtenido por los sujetos del grupo experimental en la pre y post prueba. 
En tal sentido se afirma que la aplicación de la técnica gráfico - plástica, contribuyó a mejorar la creatividad en estudiantes de instituciones unidocentes - nivel inicial, UGEL Paita - 2021

Frente a estos resultados la teoría de Guilford, considera a la creatividad, como el proceso inherente al pensar divergente, que el desarrollo de las capacidades creativas se manifiesta en todas las personas. Los resultados se confirman con el estudio de Beteta (2017) en su trabajo de investigación, sostiene que la aplicación de técnicas grafico plásticas, ayudan a mejorar la creatividad en estudiantes, complementado con los hallazgos de Parra (2016) que sostiene que la edad no es causal para desarrollar la creatividad, de la misma manera García-Pérez, (2015) manifiesta que desde temprana edad se debe estimular la creatividad. Beaudot (2016) considera que la Fluidez: es la habilidad organizar elevados números de conceptos respecto a un contenido delimitado, se demuestra cuando se le pide al niño que nombre acontecimientos, palabras, eventos. La fluidez es la cantidad de opiniones y procedimientos que posee el niño para dar solución a circunstancias presentadas, concluyendo que a más ideas mayor fluidez (Perdomo, 2017). Se corrobora con los hallazgos de Robles (2016) en su investigación realizada en Sócota, concluyendo que en las sesiones de aprendizaje se deben usar estrategias gráfico plásticas para mejorar el nivel de creatividad en los niños y niñas. por otro lado, Larraz (2021) dice que elaboración es la forma o condición para transformar fórmulas y dar solución y acción decisiva, como también es la exigencia al impulso de la creatividad. Se coincide con Mestanza (2016), cuando sostiene que las técnicas grafo plásticas permiten libertad de expresión del espíritu humano, que favorece el instinto creador del niño, porque es capaz de proponer variedad de actividades, desde su imaginación y libre albedrío, con la intención de superar reto propuestos por su tutor o docente.

\section{CONCLUSIÓN O CONSIDERACIONES FINALES}

Que la técnica gráfico - plástica mejora la creatividad en estudiantes; debido que el 100\% ubicado en nivel medio, antes de la aplicación, después del estímulo el 100\% se ubicó en nivel alto; además en la tabla 10, el nivel de significancia es $0,000<0,05$, aceptando la hipótesis de investigación y rechazando la hipótesis nula; siendo los puntajes del postest superiores al pretest, se deduce que la estrategia gráfico - plástica mejora la creatividad. 
Se ha determinado que la aplicación de la técnica gráfico - plástica mejora la fluidez en estudiantes, esto debido que el pretest el $67,86 \%$ se ubica en nivel medio, y después de la aplicación del estímulo el $100 \%$ en ubica en nivel alto. Entonces se determina que la estrategia gráfico - plástica mejora la fluidez. De la misma manera se presenta nivel de significancia es $0,000<0,05$, lo que permite aceptar la hipótesis de investigación y rechaza la hipótesis nula.

Se ha demostrado que la aplicación de la técnica gráfico - plástica mejora la originalidad en estudiantes, donde el $92,86 \%$ estaba en nivel medio del pre test y después pasan al $100 \%$ nivel alto del post test. Esto se pone de manifiesto, ya que el nivel de significancia es $0,000<0,05$, donde se acepta la hipótesis de investigación y se rechaza la hipótesis nula, por lo que se concluye que existe diferencia significativa entre el antes y después.

Se determinar que la técnica gráfico - plástica mejora la flexibilidad en estudiantes; donde del $75 \%$ nivel medio y $25 \%$ nivel bajo, antes de la aplicación; luego se ubica el $100 \%$ en nivel alto para el post test. En tal sentido se determina que la estrategia gráfico - plástica mejora la flexibilidad. Además se presenta nivel de significancia de $0,000<0,05$, aceptando la hipótesis de investigación y rechazando la hipótesis nula; por lo que se sostiene que la aplicación de la técnica gráfico - plástica mejora la flexibilidad.

Se demuestra que la técnica gráfico - plástica mejora la elaboración en estudiantes, donde se aprecia al 96,42\% ubicado en nivel medio del pre test y luego se aprecia que el 96,42\% en ubica en nivel alto en el post test. También se presenta el nivel de significancia es $0,000<0,05$, donde se acepta la hipótesis de investigación y se rechaza la hipótesis nula, sosteniendo que la estrategia gráfico - plástica mejora sustancialmente la elaboración.

\section{LISTA DE REFERENCIAS}

Ary, D.; Jacobs, L.; Razavieh, A. (1989). Introducción a la investigación pedagógica. Segunda edición. México. McGRAW-HILL

Avalos, L. (2017). El desarrollo de la creatividad en el niño de preescolar. http://200.23.113.51/pdf/24384.pdf

Barbosa, L. (2018). Fomenta la creatividad en preescolar: juega, idea, explora y dibuja. Universidad Javeriana. http://www.idep.edu.co/sites/default/files/Fomenta\%20la\%20creatividad\%20en\% 20preescolar.pdf

Beaudot, A. (2016). La creatividad. Madrid. Narcea, S. A. de Ediciones 
Bermúdez, N. \& Perreros, M. (2011). Técnicas grafo plásticas en el desarrollo de la psicomotricidad fina. (Tesis de maestría, Universidad El Milagro). Milagro, Ecuador.

Bernal, C. (2010). Metodología de la Investigación. Administración, Economía, Humanidades y Ciencias Sociales. 3ra edición. Colombia: Pearson educación.

Beteta, A. (2017). Diseño de un programa de técnicas grafico plásticas para el desarrollo de la motricidad fina en los niños de la I.E.I. $N^{\circ} 088$ José Carlos Mariátegui Castillo grande - Tingo María. http://repositorio.udh.edu.pe/123456989/505

Bonilla, E.; Rodríguez, P. (1997). Más allá del dilema de los métodos. La investigación en ciencias sociales. $3^{\mathrm{a}}$ Ed. Santafé de Bogotá, Ediciones Uniandes,

Carrasco, S. (2009). Metodología de la investigación científica. Lima, Perú: San Marcos.

CEPAL. (2015). Invertir mejor para invertir más. Financiamiento y gestión de la Educación en América Latina y el Caribe. Naciones Unidas: Santiago de Chile.

Csikszenmihalyi, M. (1998). Creatividad. El fluir y la psicología del descubrimiento y la invención. Paidós. Barcelona.

Díaz, C. (2006). La creatividad en la expresión plástica. Editorial Narcea Madrid. España.

Esquivias, S. (2005). Una evaluación de la creatividad en la Educación Primaria.http://www.revista.unam.mx/vol.1/num3/art1/

Gallego, Gil y Bedoya (2016). La expresión gráfico-plástica en la primera infancia: una alternativa didáctica y pedagógica. Revista

García-Pérez A. (2015). Creatividad en alumnos de primaria: evaluación e intervención"- Salamanca, 2015. (Tesis doctoral, Universidad de Salamanca, España)https://gredos.usal.es/handle/10366/129409

Gardner, H. (1998). A Reply to Perry D. Klein's 'Multiplying the problems of intelligence by eight'". Canadian Journal of Education 23 (1): 96-102. Doi:10.2307/1585968. JSTOR 1585790.

Hernández, R., Fernández, C., Baptista, P. (2014). Metodología de la investigación. Sexta edición. México: McGraw-Hill/Interamericana Editores.

Hernández, R.; Zapata, N.; Mendoza, C. (2013). Metodología de la investigación para bachillerato. Enfoque por competencias. México: McGraw-Hill/Interamericana Editores. 
Hernández, Zapata, Mendoza (2013). Metodología de la investigación para bachillerato. Enfoque por competencias. México: McGraw-Hill/Interamericana Editores

Joas, H. (2005). The Creativity of Action, Chicago, University of Chicago Press.

Latorre, A.; Delio del Rincón, I. Arnal, J. (1996). Bases metodológicas de la investigación educativa. Barcelona: GR92.

Marín, J. (2009). Fundamentación epistemológica para la investigación pedagógica. file://C:/Users/Computer/Downloads/Dialnet-

FundamentacionEpistemologicaParaLaInvestigacionPed-3438917.pdf

Mestanza G. (2016). Programa de técnicas gráfico plásticas para desarrollar la coordinación motora fina en los niños y niñas de la IEI $N^{\circ} 302$ de Sócota, Cutervo2016. (Tesis doctoral, Universidad César Vallejo). http://repositorio.ucv.edu.pe/handle/UCV/2105?show=full

Montealegre, R. (2016). Controversias piaget-vygotski en psicología del desarrollo Acta Colombiana de Psicología, vol. 19, núm. 1, 2016, pp. 271-283. https://www.redalyc.org/pdf/798/79845405012.pdf

Parra, M. (2016). La creatividad en la educación infantil de las instituciones educativas públicas y privadas de la ciudad de Bucaramanga. (Tesis doctoral, Universidad de Granada). https://hera.ugr.es/tesisugr/25681369.pdf

Pino, R. (2007). Metodología de la investigación. Lima, Perú: Editorial San Marcos.

Quispe, J.; Aguirre, F. (2017). La Creatividad en los Niños de Prescolar, un Reto de la Educación Contemporánea. Doi:10.15366/reice2017.15.2

Robles, N. (2016). Programa de técnicas gráfico plásticas para desarrollar la creatividad en los niños y niñas de la I.E.I. $N^{\circ} 598$ "los amautas” de Sócota, cutervo-2016. (Tesis doctoral, Universidad César Vallejo). http://repositorio.ucv.edu.pe/bitstream/handle/UCV/2493/robles_vn.pdf?sequence $=1 \&$ isAllowed $=\mathrm{y}$

Roche, J. (2017). Creativity as an exponent of contemporary social and cultural contradictions. Approach to its concept from the sociological theory. https://www.tandfonline.com/doi/abs/10.1080/03906701.2017.1411389?journalC $\underline{\text { ode }=\operatorname{cirs} 20}$

Rodríguez, Elena (2013). Pedagogía Montessori: Postulados generales y aportaciones al sistema educativo. Córdova. 
Rojas, C. (2016) ¿Qué es pensamiento crítico? Sus dimensiones y fundamentos históricofilosóficos. Universidad de Puerto Rico. Colegio Universitario de Humacao.

Romero, F. (2015). Aprendizaje significativo y constructivismo. https://www.feandalucia.ccoo.es/docu/p5sd4981.pdf

Salkind, N. (1999). Métodos de investigación. México: Prentice Hall.

Sánchez, H., Reyes, C. (1998). Metodología y diseños en la investigación científica. Lima: Perú. Editorial Mantaro.

Sawyer, R. (2016). Explain crativity: the science of human innovation. Oxford university Press, ed. New York.

Sternberg, R., Lubart, T. (1997). La Creatividad en una cultura conformista: un desafío a las masas. Barcelona: Paidós.

Swift, E. (2015). Desarrollo de la creatividad en el aula de Educación Primaria a través del proyecto artístico multidisciplinar. https://reunir.unir.net/bitstream/handle/123456789/3256/SWIFT\%20GARCIA\%2 C\%20STEPHANY.pdf? sequence $=1 \&$ isAllowed $=\mathrm{y}$

Torrance, E. (1995). ¿Por qué volar? Una filosofía de la creatividad. Norwood: Ablex.

UNESCO (2010). Hacia las sociedades del conocimiento. Informe mundial, París, Organización de las Naciones Unidas para la Educación, la Ciencia y la Cultura. http://www.flacso.edu.mx/ colaboratorio/pdf/colaboratorio_unesco.pdf.

Valderrama, S. (2013). Pasos para elaborar proyectos de investigación científica. Cuantitativa, cualitativa y mixta. Segunda edición. Lima, Perú: Editorial San Marcos. 